\title{
ELECTRIFICACIÓN RURAL DE JUNÍN CON ENERGÍAS RENOVABLES NO CONVENCIONALES*
}

\author{
Baldeón De la Rosa, Augusto ${ }^{1}$, Cairo Hurtado, Jorge ${ }^{2}$ y Torres Mayta, Pedro 3 \\ Facultad de Ingeniería Eléctrica de la Universidad Nacional del Centro del Perú
}

\begin{abstract}
RESUMEN
El trabajo de investigación tiene por objetivo evaluar la energía no convencional renovable limpia, ecológica y barata con la finalidad de emplearla para la electrificación de las localidades rurales aisladas de la Región Junín fuera del ámbito de influencia del sistema eléctrico interconectado nacional (SEIN), determinando su impacto en el medio ambiente y el bienestar de la población de las comunidades rurales, luego proponer la generación de la energía eléctrica , aprovechando la fuerza del viento y la irradiación solar. Para ello se verifica y analiza los datos proporcionados por el SENAMHI, referido a la energía eólica y solar en el Perú. Los resultados muestran que en ciertos lugares de la Región Junín es factible la construcción, montaje y posterior operación de centros de generación de energía eléctrica utilizando fuentes de energía renovables no convencional, ya que de acuerdo al mapa eólico preliminar en la sierra los promedios de velocidad del viento es de 2 a $4 \mathrm{~m} / \mathrm{s}$ y la irradiación solar en la Región Junín es de $6.78 \mathrm{Kwh}$. $/ \mathrm{m}^{2}$. En la selva el promedio de la velocidad del viento es de $2 \mathrm{~m} / \mathrm{s}$., las cuales pueden ser aprovechadas para el bombeo de agua por medio de molinos u otras aplicaciones, pudiéndose también aprovechar de esta forma la generación de energía eléctrica mediante pico centrales hidráulicas. La idea de evolucionar hacia un mundo energético diferente esta materializándose y los diferentes gobiernos deben desempeñar plenamente su papel en este nuevo proceso para convertirse en interlocutores naturales, junto a las empresas energéticas, para tratar esta problemática desde el punto de vista tanto de la demanda como de la oferta.
\end{abstract}

Palabras clave: energía solar-energíam, eólica-energía renovable, microrredes electrificación, irradiación solaraerogeneradores, acumuladores de Energía

\section{"RURAL ELECTRIFICATION WITH UNCONVENTIONAL RENEWABLE ENERGY JUNÍN"}

\begin{abstract}
This research aims to evaluate the non-conventional, $100 \%$ organic, clean, cheap, renewable energy for the purpose of electrification in the remote villages of the Junín Region, outside of reach of the national interconnected power system (SEIN), determining its impact on the environment and people's welfare in rural communities to later propose the generation of electrical power by the strength of wind and solar irradiation. This study, then, verifies and analyzes data provided by the SENAMHI, referring to wind and solar power in Peru. The results show that in some parts of the the Junín Region, it is feasible to build, install and operate centers for renewable energy electrical sources, since according to the preliminary wind map in the mountains, the average wind speed is around 2 to 4 $\mathrm{m} / \mathrm{s}$ and the solar irradiation in the Junín Region is around $6.78 \mathrm{Kwh} / \mathrm{m}^{2}$. In the Peruvian jungle, the average wind speed is $2 \mathrm{~m} / \mathrm{s}$, which can be used for pumping water by mills or other applications; this could also take the form of electrical energy generation through central hydraulic peaks. The idea of moving towards different, cleaner, energy sources is evident, and the various governments must play a key role during these processes to become natural partners with energy companies, to address this problem in terms of both demand and supply.
\end{abstract}

Key words: solar energy, heolic energy, electrification, solar irradiation, aerogenerators, energy accumulators.

\footnotetext{
* Este trabajo de investigación fue recibido el 20/03/2009 retornado para su revisión 26/10/2009 y aprobado para su publicación 16/11/2009

1Email: albaldeon@hotmail.com

${ }^{2}$ Email: jcairo@uncp.edu.pe

${ }^{3}$ Email: ptorremn@hotmail.com
} 


\section{INTRODUCCIÓN}

La energía provee servicios que son esenciales para el desarrollo humano, social y económico, como son iluminación, refrigeración, cocinado de alimentos, calefacción, acceso a información, transporte, fuerza motriz, etc. Todos ellos son servicios absolutamente necesarios para acceder a la educación, la salud, la comunicación, el transporte y los procesos productivos. La electricidad, como forma moderna de energía, es un vector esencial en la lucha contra la pobreza y es una de las condiciones necesarias para avanzar en el desarrollo, pero actualmente hay un gran número de familias que no tienen acceso a la electricidad especialmente en las zonas rurales de la Región Junín de bajos recursos económicos. La utilización de fuentes de energía renovables, ya sea de forma individual o colectiva a través de microrredes, son soluciones operativas y reales para aquellas zonas donde el coste de extensión de las redes es prohibido.La electrificación con energías no convencionales en las zonas rurales es una alternativa viable para las comunidades donde la energía eléctrica de origen convencional es inaccesible y muy costosa. En tal sentido el presente trabajo nos orienta como aprovechar el uso de energía renovable no convencional, evitando la contaminación y conservando la ecología para electrificar zonas rurales en la Región Junín.

\section{MATERIAL Y MÉTODOS}

En la distribución espacial del viento se debe tener presente la influencia de la fuerza de Coriolis. El análisis de la circulación general provee sólo indicaciones cualitativas. La situación anemológica de una localidad o de una región está influenciada por numerosos factores. En ciertos lugares el efecto del relieve puede ser predominante; se manifiesta en la formación de turbulencia, brisas de mar y tierra, brisas de valle y montaña con patrón estacional o diario.

La evaluación del viento para descubrir las características energéticas requiere la disponibilidad de datos correspondientes a un período de tiempo suficientemente largo, medidos en puntos apropiados, bien distribuidos en el área a evaluar y a alturas convenientes. La estimación del potencial del viento en áreas o sitios de interés puede realizarse mediante un procedimiento que comprende las siguientes etapas:

- Investigación preliminar del recurso sobre la base de datos climatológicos.

- Análisis detallado del recurso a partir de las observaciones tri- 0 cuatri-diurnas.

- Medición del recurso mediante una red de adquisición específica de datos.

Los datos climatológicos no son suficientes para conocer con certeza la energía disponible y el posible comportamiento de los aerogeneradores. Por lo tanto, se hace necesario efectuar un análisis detallado de las observaciones diarias obtenidas en todas las estaciones meteorológicas. De esta forma se obtienen estimaciones representativas de la energía y su potencia disponibles. Una vez obtenidos estos resultados, es necesario centrar la atención en aquellos lugares de interés que se muestren favorables para la instalación de máquinas eólicas. La selección de los lugares donde se realizarán las mediciones se efectúa tomando en cuenta la información de las estaciones convencionales más cercanas, las características orográficas, presencia de obstáculos y canalización del viento.

La forma más usual de medir la fuerza del Sol es en $\mathrm{kw} / \mathrm{m}^{2}$ de área horizontal. En el mapa de radiación solar emitida por el SENAMHI indica que la irradiación diaria media anual en la Región Junín es de 6.78 $\mathrm{Kwh} / \mathrm{m}^{2}$.

El aprovechamiento activo se puede regir bajo dos puntos de vista bien diferenciados: la conversión térmica 0 aprovechamiento del calor contenido en la radiación solar, y la conversión eléctrica o aprovechamiento de la energía luminosa (fotones), para generar directamente energía eléctrica.

\section{RESULTADOS}

En el mapa eólico preliminar, se observa que el mayor potencial eólico en el Perú se encuentra ubicado en nuestro litoral. El resultado de las evaluaciones preliminares de los años anteriores, se han registrado velocidades de viento mayores a $5 \mathrm{~m} / \mathrm{s}$, de acuerdo a 
la industria eólica estos valores son económicamente favorables. Zonas de mayor potencial eólico, desde Ica hasta Tacna por el Sur y de Ancash hasta Tumbes por el norte. En la sierra, los promedios de la velocidad de viento son de 2 a $4 \mathrm{~m} / \mathrm{s}$. En la selva: el promedio de velocidades de viento son menores 0 iguales a $2 \mathrm{~m} / \mathrm{s}$. En zonas de sierra o la selva, este potencial puede ser aprovechado en otras aplicaciones distintas a la generación de energía eléctrica así como bombeo de agua por medio de molinos u otras aplicaciones.

Tabla 1. Irradiación solar del Perú (senamhi)

\begin{tabular}{|c|c|c|c|}
\hline $\mathbf{N}^{\bullet}$ & Departamento & Estación & $\begin{array}{l}\text { RRADIACION DIARIA } \\
\text { MEDIA ANUAL } \\
\text { KWhin2 }\end{array}$ \\
\hline 59 & San Martín & Tarepoto & 4.50 \\
\hline 6 & Lambayeque & Lambaveque & 5.00 \\
\hline 61 & Ancash & Huaraz & 5.75 \\
\hline 62 & Lima & Andahuasi & 5.79 \\
\hline 63 & Junin & 1.Geofísion. Huancavo & 6.78 \\
\hline 64 & Lima & La Molina & 4.09 \\
\hline 65 & Lima & Acantarilla & 4.39 \\
\hline 66 & Lima & Sta. Rosa & 5.23 \\
\hline 67 & Cusco & Grania Kcayra & 5.28 \\
\hline$\varnothing$ & Ica & Ica & 5.27 \\
\hline$\Leftrightarrow$ & Puno & Puno & 6.80 \\
\hline 70 & Arequipa & Pampa de Maies & 5.11 \\
\hline 71 & Arequipa & Characato & 7.09 \\
\hline 72 & Moquequa & Pta Coles & 4.86 \\
\hline 73 & Moquequa & Moquequa & 6.13 \\
\hline 74 & Tacna & Calana & 5.44 \\
\hline 75 & Arequipa & Pampa Blanca & 4.27 \\
\hline 76 & Arequipa & Haciendita & 5.23 \\
\hline
\end{tabular}

\section{DISCUSIÓN}

En general, cuando se trata de zonas empobrecidas y aisladas, el uso de energía generada mediante sistemas renovables aislados suele ser la mejor solución Sin embargo, hay que considerar que la tecnología es solo una parte de la solución del problema, la formación en el uso de las energías es esencial en la sostenibilidad de un proyecto y en el uso productivo del mismo. Para que se den estas condiciones, es imprescindible la "alfabetización energética" de la población en general y una sencilla formación especializada para algunos grupos o personas necesarios para el mantenimiento y gestión de las instalaciones.

Los trabajos realizados en el presente estudio indican que es conveniente la instalación de un sistema híbrido solar y eólico- en vez de utilizar sólo una de las fuentes alternativas debido a que se demuestra una notable economía en el dimensionamiento de equipos y en el banco de baterías. La energía solar y eólica, en consecuencia, podrían ser utilizadas para dar solución al problema de iluminación y alimentación a equipos electrodomésticos y de comunicación, con lo que se obtendrá un impacto favorable en el proceso de adelanto tecnológico de las comunidades.

\section{CONCLUSIONES}

- Como el resto de energías renovables, la eólica y la solar son fuentes de electricidad limpia, inagotable y autóctona, lo cual representa importantes ventajas ambientales y socioeconómicas. Esto no quita que, como cualquier otro sistema de generación eléctrica, esta fuente de energía tenga impactos negativos sobre el medio ambiente; no obstante, hoy en día el balance resulta mucho más que positivo en comparación con las energías tradicionales que emplean combustibles fósiles o radiactivos.

- Después de los análisis en el presente trabajo se ha llegado a la conclusión que es conveniente la instalación de un sistema híbrido solar y eólico para la electrificación de las comunidades aisladas de la Región Junín.

- A diferencia de los sistemas de generación tradicionales, la energía eólica no genera residuos peligrosos radiactivos ni vierte a la atmósfera dióxido de carbono (CO2), dióxido de azufre (S02), óxidos de nitrógeno (NOx) o partículas sólidas. El S02, el NOx y los metales pesados son sustancias contaminantes con una importante incidencia en la salud de los ciudadanos y el medio ambiente, mientras que el C02 es uno de los principales gases de efecto invernadero causantes del calentamiento de la Tierra, cuya reducción y control es uno de los mayores desafíos actuales de la humanidad.

- La energía solar es uno de esos recursos renovables que nos regala la naturaleza a cada instante, y lo que es igual de importante, una fuente de energía que no daña el entorno en que vivimos.

- La energía solar también contribuye eficazmente a la reducción de emisiones de C02, responsables del calentamiento global del planeta. Se 
calcula que con el uso de una instalación solar para la producción de agua caliente sanitaria, una familia puede evitar, la emisión de una tonelada de C02 al año.

\section{LITERATURA CITADA}

Araujo, T. S, Simoes, F. N; Silva, M. 1987. Projeto e estudo de un gerador de pequeno porte .En: IV Congreso Brasileiro de Energía. R. J., Brasil, Vol. 1/3, 1987: 267-276.

Barros V. R; Estevan, E. 1983. Cálculo de la potencia eólica en base a series cortas de viento. En: Meteorológica, Vol. XIV No 1 y 2: 495-503.

Grossi Gallegos H, Lopardo, R, Atienza, G, García, M, Peralta, M. 1986. Distribución de la energía solar en la región meridional de América del Sur. En: Memoria Técnica del $5^{\circ}$ Congreso Latinoamericano de energía solar, Tomo I: 1-11, Valparaíso, Chile.
Hogtert C. N. 1986. Estudio comparativo de la energía efectiva producida mediante aerogeneradores. En: Memoria técnica del 5to. Congreso Latinoamericano de energía Solar, Tomo II : H1-H13, Valparaíso, Chile.

Ibacache M. E. 1986. Las energías solar y eólica y las posibilidades de financiamiento internacional en América Latina. Actas del 5to. Congreso Latinoamericano de energía Solar, Valparaíso, Chile.

OLADE/PNUMA. 1980. Alternativas energéticas en América Latina. Estudio de capacidades para el uso de fuentes no convencionales de energía. Quito, Ecuador, 1979: 1-407. OLADE. "Prospección, evaluación y caracterización de la energía eólica". Documentos OLADE, N10, Quito, Ecuador.

Seguel R. C; Vera, V. R. 1986. El viento una alternativa en sectores rurales aislados. En: Memoria Técnica del 5to. Congreso Latinoamericano de Energía Solar. Tomo II, Valparaiso, Chile: H66-H76. 